\title{
PLASMA ARMATURE CHARACTERIZATION MEASUREMENTS PERFORMED AT THE UNIVERSITY OF TEXAS
}

\author{
By: \\ D. A. Weeks \\ R. C. Zowarka, Jr. \\ J. R. Uglum
}

Seventh IEEE Pulsed Power Conference, Monterey, CA, June 11-14, 1989, pp. 632-636

Center for Electromechanics

The University of Texas at Austin

PRC, Mail Code R7000

Austin, TX 78712

(512) 471-4496 


\title{
PLASMA ARMATURE CHARACTERIZATION MEASUREMENTS PERFORMED AT THE UNIVERSITY OF TEXAS
}

\author{
Center for Electromechanics \\ The University of Texas at Austin \\ Balcones Research Center \\ Building 133 \\ Austin, Texas 78758-4497 \\ D. A. Weeks and R. C. Zowarka \\ and \\ J. R. Uglum \\ Austin Research Associates \\ 1901 Rutland \\ Austin, Texas 78758
}

\begin{abstract}
The Center for Electromechanics at The University of Texas at Austin (CEM-UT) and Austin Research Associates (ARA), in a cooperative effort, have performed a series of high performance railgun experiments in order to measure plasma armature characteristics. The tests were performed at CEM-UT using a $1 \mathrm{~m}$ long, $12.7-\mathrm{mm}$ square bore railgun. Twenty experiments were performed. In each experiment plasma pressure, arc voltage, breech voltage, arc spectrum, gun current, and magnetic probe (B-dot) signals were recorded. Glass filled polycarbonate insulators and molybdenum rails were used in all experiments. Gun currents ranged from 150 to $450 \mathrm{kA}$. Experiments were performed primarily at atmospheric pressure. The projectile in most experiments was a polycarbonate cube approximately $2.5 \mathrm{~g}$ in mass. A few experiments were performed in vacuum with a free running arc. In all cases, the arc was initiated by electrically exploding an aluminum foil fuse.
\end{abstract}

Experimental measurements made with a piezoelectric pressure transducer indicates that pressure profile is time correlated with the armature B-dot profile. The measured parabolic pressure profile is consistent with that expected by theoretical models. Peak pressures recorded were between 30 MegaPascals $(\mathrm{MPa})(4,300 \mathrm{psi})$ and $200 \mathrm{MPa}$ $(30,000 \mathrm{psi})$ and correlated well to the pressure determined by dividing the Lorentz force (calculated from the gun current when the projectile passes the transducer) by the bore cross sectional area. Also of interest is that in-bore intensity measurements made with a PIN diode indicate light emission time is comparable to the pressure profile duration.

Analysis of the emitted spectrum shows continuum radiation with strong absorption lines that are associated with neutral molybdenum and neutral aluminum.

Plasma resistivity in the transducer region was calculated from the gun current, muzzle volts, bore area, and the armature length as determined by the B-dot probes. The resistivity calculated appears to be consistent with previously published articles indicating that plasma resistivity is inversely proportional to the fourth root of the plasma's pressure.

Due to the quick turn around afforded by the modified railgun breech, the same experimental parameters were repeated on several tests producing results that typically correlated better than $5 \%$.

\section{Introduction}

High current density operation is motivated by the desire to keep the gun structure compact. Simple considerations show that for a given gun length, increased velocity performance requires higher operating pressure on the projectile; high pressure in the plasma then dictates high current density. For experimental systems being studied, a pressure of $200 \mathrm{MPa}(30,000 \mathrm{psi})$ is the design goal. In this work it has been attempted to characterize the resistivity of plasma armatures at these high pressures. The results tend to support physical models used to compute this transport coefficient.
The experimental configuration chosen for use was notivated by a desire to test the physical assumptions underlying models of plasma armatures. In particular, if radiation transport is dominant within the bulk plasma, then local thermodynamic equilibrium obtains, and the Saha relations governing ionization states are valid. This in turn leads to a prediction that the plasma conductivity should have a simple scaling with pressure; temperature effects should be negligikle (in bulk).

\section{Plasma Armatures}

A plasma armature can be considered as a transclucer which converts electromagnetic momentum flux into thermodynanic pressure; this pressure then accelerates both armature and projectile. The pressure profile within the plasma will peak at the back face of the projectile; in general, the pressure then monotonically falls to zero at the end of the armature. It is generally assumed that this profile is quasi-static in time, with magnetosonic waves providing temporal structure. Details of the equilibrium pressure profile will depend on the the:mophysical properties of the plasma, in particular on its resistivity.

For advanced hypervelocity railguns, peak operating, pressure of $200 \mathrm{MPa}(30,000 \mathrm{psi})$ or more is required. In this regime, theoretical estimates of plasma temperature and density indicate that the plasma parameter, that is the number of particles in a Debye sphere, is of order one. This entails a re-examination of the assumptions entering into calculations of plasma properties, in particular the resistiv ty.

At low pressure, plasma resistivity is adequately cescribed by using the conventional Spitzer formula applied to a mul:i-ionization state atomic species. This model has been extended by Aus:in Research Associates, and also Kovitya[1], to the pressure regime anticipated for operational railguns. Calculations show that the resistivity for metallic plasmas display rather interesting features. For jressures in the 20 to $200 \mathrm{MPa}(3,000$ to $30,000 \mathrm{psi})$ range, the resistivity shows a rapid decrease with temperature up to about $20,000 \mathrm{~K}(\sim 2 \mathrm{e} / \mathrm{l})$, and then flattens in its dependence on temperatures up to 60,000 to $70,000 \mathrm{~K}$ (6 to 7 $\mathrm{eV})$. The resistivity in this range also shows weak pressure dependence. To a good approximation, the formula

$$
\eta=\frac{\text { Constant }}{\mathrm{T}^{1 / 4} \mathrm{p}^{1 / 4}}
$$

fits the theoretical results very accurately in the rarige

$$
\begin{array}{ll}
2 \leq \mathrm{T} \leq 6 & \text { electron volts } \\
20 \leq \mathrm{P} \leq 200 & \text { MegaPascal }
\end{array}
$$

Further, because of the similarity in ionization potentials for metal atoms, this equation should hold for any high atomic number plasma.

Armature structure is expected to depend primarily on pressure. A simple model can be developed using this assumption, as shown in Reference 2. The result indicates that if neglect of :emperature 
dependence is justified, then the plasma resistivity can be measured easily and the results used to cross check the pressure dependence. Further, the peak plasma pressure is directly proportional to the square of the driving current, allowing easy adjustment of pressure by variation of drive current.

This feature of expected plasma armature resistivity was addressed in our experimental program. Several pressures in the range from 30 to $200 \mathrm{MPa}(4,500$ to $30,000 \mathrm{psi})$ were selected and the resistivity inferred from the diagnostics used. The results given below show good agreement with model predictions.

During the course of experimental design, the capacitive store facility at CEM-UT was identified as the most appropriate test bed for carrying out these measurements. A joint program was initiated by ARA and CEM-UT. Funding for CEM-UT staff members, and facility costs, was provided by a grant from the Texas Atomic Energy Research Foundation.

\section{CEM-UT Capacitor Bank Railgun Facility}

The desire to vary operating pressure over a large range by control of the driving current was the primary reason for utilizing the CEM-UT capacitive store facility for the experiments. In addition, the availability of the railgun, diagnostic probes, screen room, and data acquisition devices allowed the experimental program to proceed at a rapid and cost effective rate.

The capacitive store facility shown in Figure 1 consists of 14 capacitive/inductive store modules which can be charged to $10-\mathrm{kV}$ peaks. Each module has a series inductance of approx:mately $2.5 \mu \mathrm{H}$ and a series resistance of about $3 \mathrm{~m} \Omega$. The nominal capacitance of each module is 1 to $2 \mathrm{mF}$. The discharge of each module is controlled by a triggered ignitron so that the current waveform shape may be controlled by varying the timing sequence of the stores. A self-triggered ignitron on each module crowbars the capacitors to prevent voltage reversal following peak current. Each module is connected to a common buswork which feeds the breech of the railgun. For experimental planning a simulation code is used to select the charging voltage and module sequencing required to achieve the desired gun current and pulse duration. The current profile was designed to provide constant acceleration of the projectile in the region which the measurements took place so that the average velocity computed from adjacent B-dots would be valic.

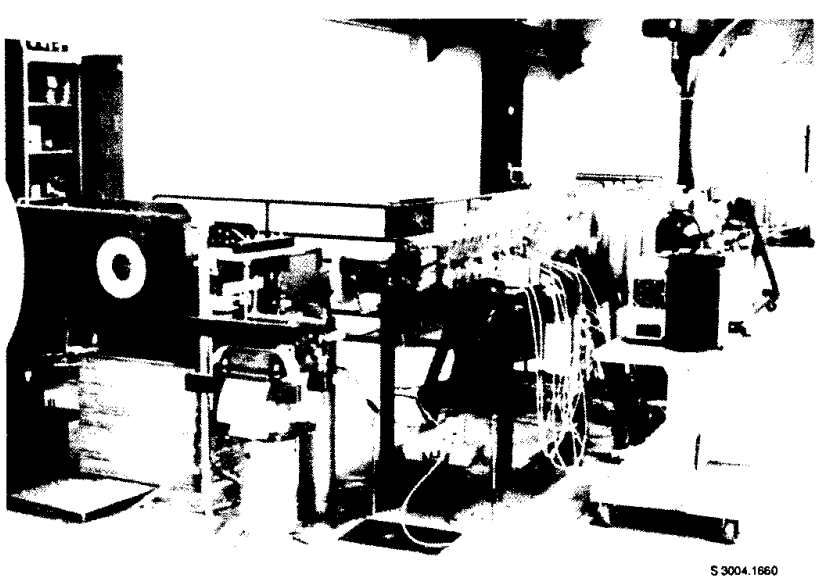

Figure 1. CEM-UT capacitor bank railgun facility

To reduce damage to the muzzle of the gun and to speed up turn around, an ignitron crowbar switch was incorporated at the breech of the railgun. It was sequenced to close when the projectile had traveled approximately three quarters of a meter.

\section{Railgun Configuration}

The gun structure selected for use, shown in Figure 2 was $1 \mathrm{~m}$ long and had a $12.7-\mathrm{mm}$ square bore. Solid molybdenum rails and $40 \%$ glass- filled polycarbonate sidewalls were used for longevity. To improve sealing the rails and insulators were assembled using fiber re-enforced epoxy impregnated tape and then cured in a vacuum bag at $200^{\circ} \mathrm{F}$ for 4 hr. The assembled gun components were pressed into a filament wound fiberglass tube and then clamped in a bolt-up square steel containment frame.

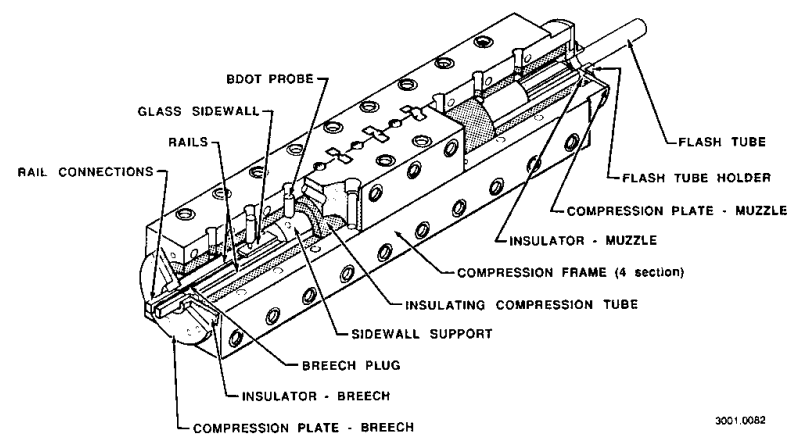

Figure 2. ARA experimental railgun configuration

The bore surfaces were initially honed parallel to within \pm 0.05 $\mathrm{mm}( \pm 0.002 \mathrm{in}$.) using a diamond grit hone on the rail surface and a 30 micron grit sandpaper hone on the insulator surface. The bore dimensions were measured using an air gauge and precision gauge blocks. Initially the rail to rail separation was $12.66 \mathrm{~mm}(0.498 \mathrm{in}$.) and the insulator to insulator separation was $12.84 \mathrm{~mm}(0.505 \mathrm{in}$.). After 18 experiments the dimensions had increased to $12.84 \mathrm{~mm}(0.505 \mathrm{in}$.) rail to rail and 14.23 $\mathrm{mm}(0.560 \mathrm{in}$.) insulator to insulator.

Following the initial honing of the gun, its inductance gradient was measured using an Electro Scientific Industries Video Bridge operating at $100 \mathrm{kHz}$ frequency. A least squares data fit of inductance vs. position yielded an inductance gradient of $0.34 \mu \mathrm{H} / \mathrm{m}$.

Polycarbonate cubes approximately $12.7 \mathrm{~mm}(0.500$ in.) square and approximately $2.5 \mathrm{~g}$ in mass were employed for projectiles. The breech buswork was configured so that the monolithic fuse holder/breech plug could be removed and replaced without disassembly. A $0.15 \mathrm{~mm}$ ( 0.006 in.) thick aluminum foil was attached to the breech plug to initiate the arc. A $2 \mathrm{~cm}$ gap was left between the projectile and breech plug to reduce the thermodynamic velocity imparted to the projectile associated with the ohmic melt and vaporization of the foil fuse.

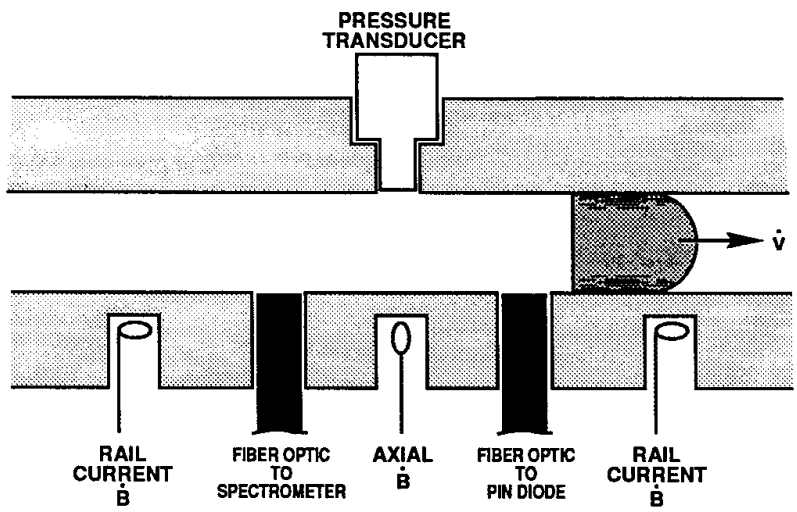

3001.0092 


\section{Diagnostic System}

In addition to standard railgun instrumentation(B-dot probes, muzzle and breech volts, and Rogowski coils) a piezoelectric pressure transducer, a PIN diode, and a spectrometer were used to measure the plasma characteristics during experiments. These additional diagnostic devices were located $27.5 \mathrm{~cm}$ (10.82 in.) from the breech of the gun opposite B-dot probe \#3 as seen in Figure 3. This location was chosen because the armature/projectile velocity would be about $1 \mathrm{~km} / \mathrm{s}$, thus minimizing viscous drag effects, and allowing the driving current to be tailored to minimize muzzle arcing.

The piezoelectric pressure transducer measures the armature pressure directly during flyby. The axial B-dot located at position \#3 was used to determine the time duration of the current-carrying portion of the armature. Radially oriented B-dot probes, spaced $10 \mathrm{~cm}$ (2.5 in.) on either side of the test location were used to determine the average velocity at the test location. Gun current and muzzle voltage traces determined the armature resistance as a function of time. Finally, two fiberoptic probes provided direct bore surface access for monitoring light output and spectral characteristics.

A piezoelectric pressure transducer was mounted opposite magnetic probe location \#3 to directly measure armature pressure during fly-by. The PCB Model 108A has a light-tight active area, a $2 \mu \mathrm{s}$ risetime, and a linear pressure range up to $550 \mathrm{MPa}(80,000 \mathrm{psi})$. It was recess mounted in the insulator sidewall; the active area within $0.2 \mathrm{~cm}$ $(0.08 \mathrm{in}$.) of the bore surface and was coupled directly to the armature plasma through a $0.2 \mathrm{~cm}(0.08 \mathrm{in}$.) hole drilled through the sidewall.

Because the transducer directly contacted the plasma, its output was electrically isolated by a fiberoptic link, utilizing a solid state laser transmitter/receiver circuit. The gain and frequency response of this analog fiberoptic transmission system was measured before each gun shot by use of a calibrated square wave signal generator.

The transducer was calibrated by the manufacturer. The sensitivity was measured to be $100 \mathrm{MPa} / \mathrm{V}(15,000 \mathrm{psi} / \mathrm{V})$.

The light output from the plasma armature surface was detected using a PIN diode and time-integrated spectral information from the arc's surface was recorded on a spectrometer. The PIN diode and spectrometer were connected to the gun bore with a $0.1 \mathrm{~cm}(0.040 \mathrm{in}$.) diameter graded index fused silica fiber which was $2-\mathrm{m}$ long. The fibers were epoxied into the gun flush with the insulator surface approximately $2.5 \mathrm{~cm}$ (1.0 in.) either side of B-dot location \#3.

The spectrometer used was a Minute Man Labs of Acton, Massachusetts Model 205. It has an adjustable wavelength center and lithium fluoride coated aluminum mirrors. After each experiment known spectral lines were exposed on the film above and below the recorded data using a mercury light source. The light was coupled to the spectrometer by a $2 \mathrm{~m}$ (79 in.) long length of fused silica fiber optic to verify fiber transmission over the selected wavelength range.

The PIN diode was used to verify that the spectral information recorded was due to the armature fly-by and that another spectral source was not present.

\section{Shot Summary}

A total of 19 experiments were performed. The shot summary in Table 1 lists some of the relevant parameters of each test.

Useful data was obtained on all but the first experiment. Problems were discovered with the fiberoptic links during the firs three experiments. The hot plasma could apparently cause therma stressing and fracture of the fiber optic. Prior to Shot \#4 and subsequently thereafter, both fibers were removed, cut and polished and reseated before each test. Even then debris blockage or thermal fracture often abruptly terminated the PIN diode signal. Similar problems with the other fiberoptic channel caused varying exposure of the spectrometer film. However, general spectral features remained the same regardless of the exposure.

Pressure transducer signals were lost during the first three tests however useful data was recorded on shots 4 through 13 . Prior to test \#14 it was determined that the integrated amplifier in the transducer had failed. The transducer was sent back to the manufacturer for repair and calibration. It was not returned prior to the completion of the last six tests.

Table 1. Shot summary

\begin{tabular}{|c|c|c|c|c|c|}
\hline \multirow[b]{2}{*}{ Shot $\$$} & \multirow[b]{2}{*}{ Date } & \multirow{2}{*}{$\begin{array}{c}\text { Projectlle } \\
\text { mass (grams) }\end{array}$} & \multirow{2}{*}{$\begin{array}{c}\text { Center Wavelength } \\
\text { of Spectrometer }\end{array}$} & \multicolumn{2}{|c|}{ Stores } \\
\hline & & & & ;il used & Charge $\mathrm{kV}$ \\
\hline 1 & $09 / 16$ & 2.49 & 3,250 & 6 & 7 \\
\hline 2 & $09 / 20$ & 2.50 & 3,250 & 6 & 7 \\
\hline 3 & $09 / 23$ & 2.62 & 3,900 & 5 & 7 \\
\hline 4 & $09 / 23$ & 2.55 & 3,900 & 5 & 7 \\
\hline 5 & $09 / 26$ & 2.55 & 3,900 & 5 & 7 \\
\hline 6 & $09 / 26$ & 2.56 & 3,900 & 5 & 7 \\
\hline 7 & $09 / 27$ & 2.55 & 3,900 & 5 & 7 \\
\hline 8 & 10,05 & 2.58 & 4,200 & 6 & 5 \\
\hline 9 & $10 / 06$ & 2.60 & 4,200 & 6 & 5 \\
\hline 10 & $10 / 11$ & 2.60 & 4,200 & 6 & 5 \\
\hline 11 & $10 / 11$ & 2.60 & 4,200 & 6 & 5 \\
\hline 12 & $10 / 18$ & 2.64 & 3,600 & 4 & 9 \\
\hline 13 & $10 / 79$ & 2.64 & 3,600 & 4 & 9 \\
\hline 14 & $10 / 20$ & 2.65 & 3,600 & 6 & 9 \\
\hline 15 & 10/21 & 2.65 & 3,600 & 6 & 9 \\
\hline 16 & $10 / 21$ & 2.65 & 3,800 & 6 & 9 \\
\hline 17 (Nac.) & $11 / 01$ & 0.00 & 3,900 & 2 & 7 \\
\hline 18 (Vac.) & $11 / 04$ & 2.68 & 3,900 & 6 & 9 \\
\hline 19 & $11 / 28$ & 2.72 & - & 4 & 9 \\
\hline
\end{tabular}

Prior to each test, the pressure transducer was removed from the gun, cleaned, and then coated with a thin layer of sillicon va:zum grease for thermal protection.

\section{Experimental Observations}

The plasma armature's resistance, neglecting effect's of any rail interface drop, was determined by dividing the experimentally measured muzzle voltage by the gun current. Typical gun cu rent and arc voltage signals are presented in Figure 4. To determine the plasma arc's resistivity it was necessary to make assumptions or measurements of the current density in the armature. A direct determination of the true current density profile appears to be difficult. Since the observed axial B-dot probe signatures are consistent with a reasonably uniform current density profile a uniform current density was used as the basis of the resistivity calculations. (This does not prove that it is the only distribution which will yield the observed signatures).

Given that the uniform current density assumption is plausible, the resistivity can be calculated from the previously determined resistance, the bore geometry, and the armature's length.

The average velocity between B-dot's \#2 and 4 was calculated and then multiplied by the time interval determined from the zero crossings of the time derivative B-dot \#3, (see Figure 5 to dittermine the length of the current carrying plasma). The plasma arc's length was then used to compute it's resistivity.

A typical pressure transducer signal is shown in Firyre 6. The abrupt rise in pressure aligns well with the initial peak in the axial Bdot probe located opposite the transducer. The pressure cilculated by dividing the Lorentz Force by the bore's cross sectional area agrees with the peak value measured within $10 \%$. It is believed that the initial negative spike in the trace is due to an accelerometer effect caused by the gun frame reacting to the rail to rail repulsion forces of the gun. The source of the observed pressure oscillations is not clear. Sorne of it may be associated with the waves generated during the fill time of the cavity. Alternately, the oscillations could be due to magnetosonic waves within the plasma. A similar structure is seen in the PIN diode signal shown in Figure 7.

The primary purpose of the pressure transducer was to verify that the plasma pressure peak was equal to that calculated from the Lorentz force divided by the bore cross sectional area. This calculated peak pressure was then used for the purpose of determining the relationship between the plasma's resistivity as a function of its pressure. 

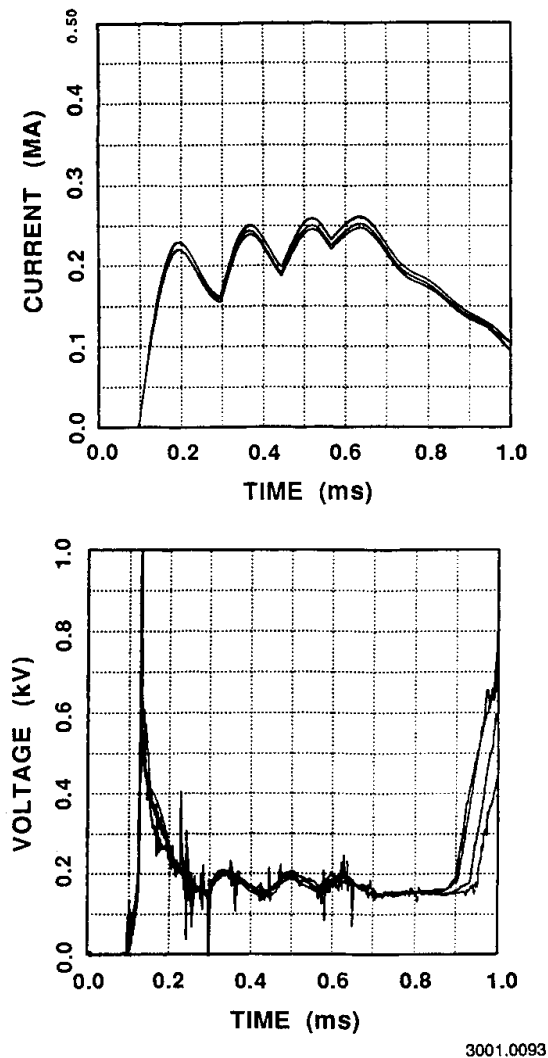

Figure 4. Typical gun current and arc voltage signals
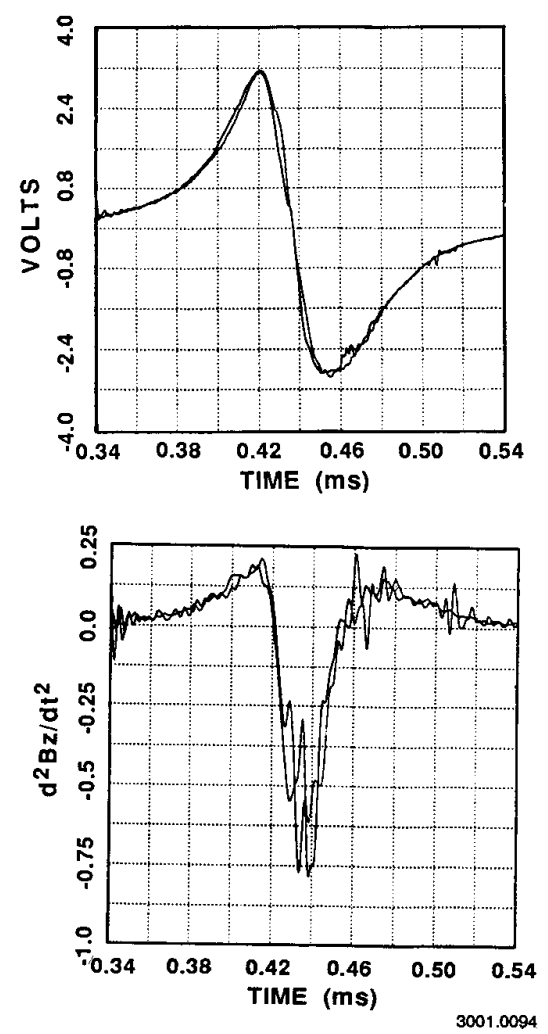

Figure 5. Derivative of axial Bdot for armature length determination

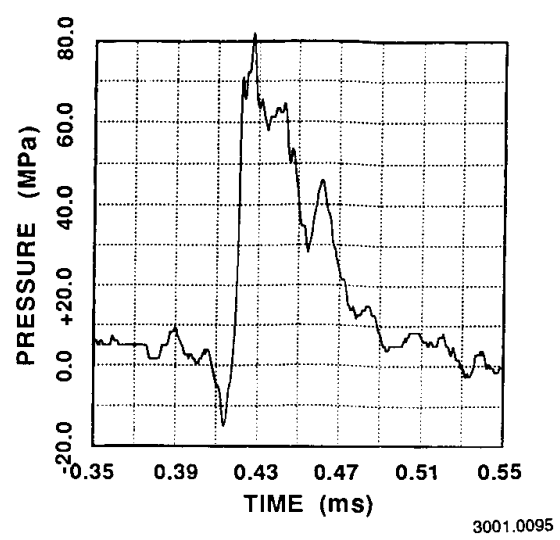

Figure 6. Typical pressure transducer signal

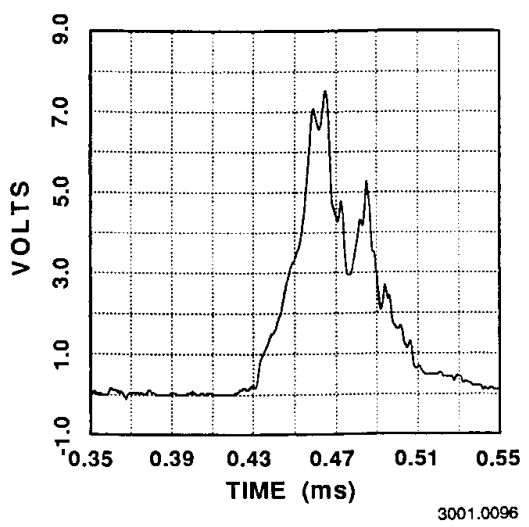

Figure 7. Typical PIN diode signal

Plasma resistivity as a function of pressure is plotted in Figure 8. Because of the finite time duration of the armature, its impedance was taken as the average value of muzzle voltage divided by gun current during this interval. As previously the mentioned the armatures length was determined by multiplying its velocity times its time duration as determined by the B-dot probes.

The solid curve corresponds to the scaling relation:

$$
\eta=\frac{\mathrm{C}_{0}}{\mathrm{P}^{.25}}
$$

where

$$
\mathrm{C}_{0}=\text { constant }
$$

The present data suggests that the resistivity scaling is roughly:

$$
\eta=\frac{3.5}{\mathrm{P}^{\prime} .25} \mathrm{~m} \Omega-\text { centimeters }
$$

where

$$
\mathrm{P}^{\prime}=\mathrm{P} / 100 \mathrm{MPa} .
$$

As a consistency check on the velocities inferred from the magnetic probes which were used to compute the plasma armatures length, predicted railgun performance and measured railgun performance for experiment \#4 are compared in Figure 9.

To insure that spectral emission recorded on the spectrometer occurred at a time consistent with the armature passing, PIN diode signal was recorded during the experiment. In Figure 7 similarity is seen in oscillation structure of PIN diode and that recorded by pressure transducer in Figure 6. 


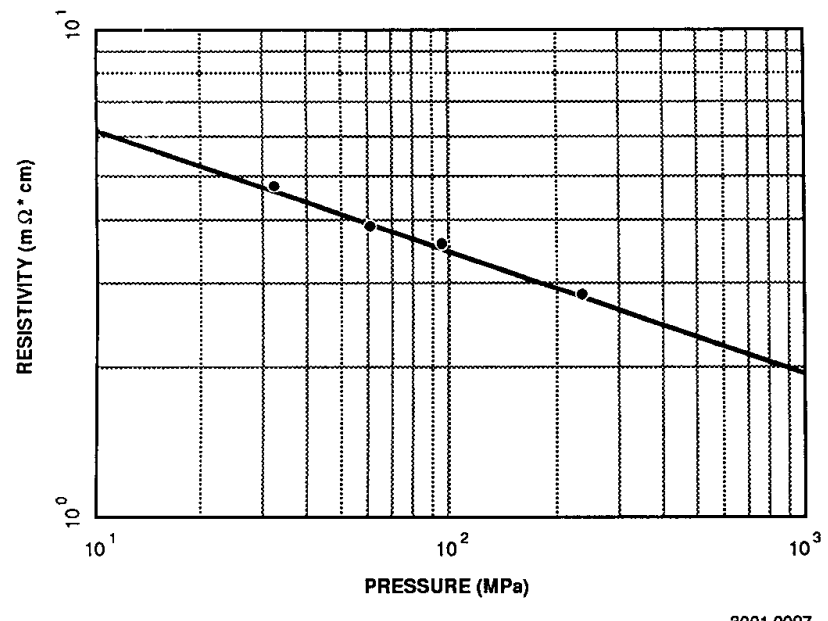

Figure 8. Experimental resistivity data

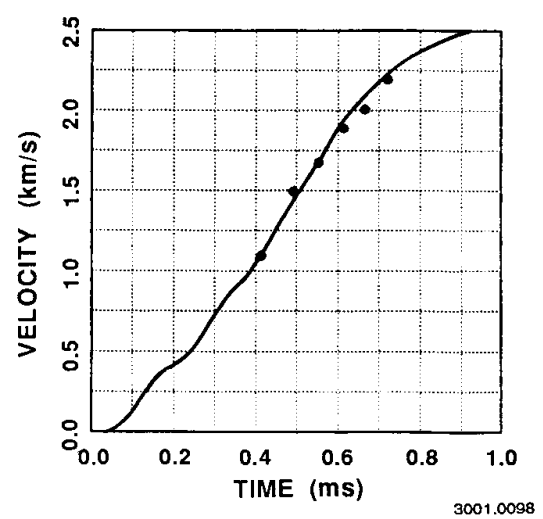

Figure 9. Predicted and experimentally measured velocity

On approximately $60 \%$ of the experiments, useful timeintegrated spectrums were obtained. The central wavelength of the spectrometer was varied from 3200 to $\mathbf{4 2 0 0}$ Angstroms during the course of the experiments. The results indicated very little light emission below 3200 Angstroms. For longer wavelengths, continuum emission, with strong absorption lines was consistently observed. Figure 10 shows a microdensitometer scan of the recorded spectral emission from experiment \#8. The absorption lines clearly show the presence of neutral molybdenum, calcium, and aluminum. Since the fiber optic was placed in the sidewall, midway between the rails, the presence of molybdenum in the spectral data suggests that ablation of rail material has occurred. It also suggests that bulk flow within the armature is occurring.

\section{Conclusions}

The plasmas resistivity assuming a uniform current distribution in the armature and neglecting rail interface voltage drop was measured over a pressure range of 30 to $200 \mathrm{MPa}(4,500$ to $30,000 \mathrm{psi})$. The resistivity calculated appears to be consistent with previously published articles indicating that plasma resistivity is proportional to the multiplicitive inverse of the fourth root of the plasma's pressure.

Analysis of the emitted spectrum shows continuum radiation with strong absorption lines that are associated with neutral molybdenum, neutral aluminum, and neutral calcium. Since, the fiber optic was placed in the sidewall, midway between the rails, the presence of molybdenum in the spectral data suggests that ablation of rail material has occurred. It also suggests that bulk flow within the armature is occurring.

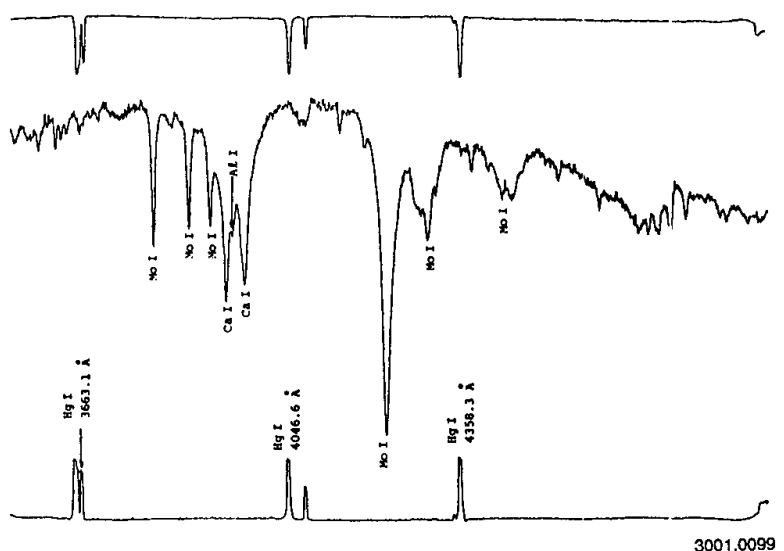

Figure 10. Microdensitometer scan of shot \#8 spectrur.

\section{Acknowledgements}

Funding for this research was provided by the Texas Atomic Energy Research Foundation. Special thanks to the effirts of the students, technicians, and engineers who performed the experiments

\section{$\underline{\text { References }}$}

1. P. Kovitya, "Physical Properties of High-Pressure Plasmas of Hydrogen and Copper in the Temperature Range 5,000 - 6,000 ${ }^{\circ} \mathrm{K}$," IEEE Transactions on Plasma Science, 6 , p.13, 1985.

2. J. Uglum, "Velocity Skin Effect with Plasma Armatures," ?'roceedings 4th Electromagnetic Launch Technology Conference, $1 \subseteq 88$, Austin, Texas. 